\title{
The Genetics of Sex: Exploring Differences
}

\author{
Michelle N. Arbeitman, ${ }^{* 1}$ Artyom Kopp, ${ }^{\dagger}$ Mark L. Siegal, ${ }^{\star}$ and Mark Van Doren ${ }^{\S}$ \\ *Department of Biomedical Sciences, College of Medicine, Florida State University, Tallahassee, Florida 32306, `Department of \\ Evolution and Ecology, University of California, Davis, California 95616, ${ }^{\ddagger}$ Center for Genomics and Systems Biology, \\ Department of Biology, New York University, New York, New York 10003, and §Biology Department, Johns Hopkins University,
} Baltimore, Maryland 21218

In this commentary, Michelle Arbeitman et al., examine the topic of the Genetics of Sex as explored in this month's issues of GENETICS and G3: Genes|Genomes|Genetics. These inaugural articles are part of a joint Genetics of Sex collection (ongoing) in the GSA journals.

EX differences affect nearly every biological process. These differences may be seen in obvious morphological traits, such as deer antlers, beetle horns, and the sexspecific color patterns of birds and butterflies. Reproductive behaviors may also be quite different between the sexes and include elaborate courtship displays, parental care of progeny, and aggressive or territorial behaviors. Beyond what meets the eye, sex differences are also pervasive in subcellular processes such as meiosis, recombination, gene expression, and dosage compensation. Sex differences are not only the domain of multicellular organisms - distinct sexes are present in most singlecell eukaryotes.

The way in which sex differences evolve and contribute to biological diversity has been studied at all levels of biological organization, from molecules and cells to populations and macroevolutionary lineages. Genetic research has focused on many questions, including characterizing the regulatory hierarchies that specify sex differences during development, determining the molecular basis for the evolution of sexspecific traits, and understanding the mechanisms of dosage compensation of sex chromosomes. With the recent advent of inexpensive sequence information and other new tools, geneticists from many disciplines are able to address questions of sex-specific biology in a much wider range of organisms and gain insight into problems for which only theoretical models

Copyright @ 2014 by the Genetics Society of America doi: 10.1534/genetics.114.165456

${ }^{1}$ Corresponding author: College of Medicine, Florida State University, 1115 W. Call St., Tallahassee, FL 32306. E-mail: michelle.arbeitman@med.fsu.edu previously existed. The fundamental genetic differences between the sexes and how they arise continue to fascinate biologists, and the results from genetic explorations of these topics are featured in an ongoing collection of articles published in GENETICS and G3: Genes|Genomes|Genetics.

The inaugural articles address some of these topics. Two studies focus on the biology of reproduction: the transition from predominantly sexual reproduction to asexuality in fungi (Solieri et al. 2014) and self-incompatibility in plants (Leducq et al. 2014). Two other articles examine differences in the evolution of sex chromosomes: Blackmon and Demuth (2014) look at the evolutionary turnover of sex chromosomes in beetles and Kirkpatrick and Guerrero (2014) use the recombining region of sex chromosomes to measure the strength of sexually antagonistic selection. Finally, two studies focus on gametogenesis-sex differences in meiosis in Caenorhabditis elegans (Checchi et al. 2014) and the specification and development of germinal cells in maize (Zhang et al. 2014).

\section{Genetic Sex Determination Occurs in Different Ways with a Myriad of Outcomes}

Many different genetic mechanisms of sex determination have been discovered in nature and studied deeply, using molecular-genetic tools (reviewed in Cline and Meyer 1996; Marin and Baker 1998; Zarkower 2001; Williams and Carroll 2009; Charlesworth and Mank 2010; Gamble and Zarkower 2012; Hughes and Rozen 2012). In humans, a dominant male-determining gene is present on the Y chromosome, whereas in Drosophila melanogaster the dose of $\mathrm{X}$ chromosomes is the primary determinate of sex, and in C. elegans sex is determined by the ratio of $\mathrm{X}$ chromosomes to autosomes. The outcomes of these pathways are also different: males and females are the result in humans and D. melanogaster vs. males and hermaphrodites in $C$ elegans. 
In the yeast Saccharomyces cerevisiae (reviewed in $\mathrm{Ni}$ et al. 2011) diploid $\mathbf{a} / \alpha$ organisms are derived by the fusion of opposite-sex, a and $\alpha$ haploids that are able to switch genetic material from one of two silent mating-type loci (HMR and $H M L$ ) to the active MAT locus through gene conversion initiated by the $\mathrm{HO}$ endonuclease. In plants that are obligate outcrossers, self-incompatibility (SI) loci prevent productive self-fertilization, thereby effectively creating many mating types (reviewed in Barrett 2002).

Solieri et al. (2014) examined the genetic basis of mating in the yeast Zygosaccharomyces sapae, which typically lives in osmotically stressful environments, is often the cause of food spoilage and displays predominantly clonal reproduction. $Z$. sapae is closely related to other species that reproduce sexually, leaving open questions of whether $Z$. sapae has the genes that normally determine sex and what factors promoted the transition toward asexuality in this lineage. The $Z$. sapae genome indeed contains genes homologous to those involved in mating-type determination in related yeast species, but it is unusual in containing three different versions of the $\alpha$ mating-type locus and two $\mathrm{HO}$ endonuclease genes, in addition to one version of the a mating-type locus. The identification of the a $\alpha \alpha \alpha$ genotype leads the authors to propose that the stressful conditions in which this yeast normally finds itself could promote genome instability, with the sex chromosome perhaps being a hot spot for recombination and mutation. This genome instability in turn would produce mating-type imbalances that could disfavor or entirely prevent sexual reproduction, thereby contributing to genetic diversity in mating strategy among Zygosaccharomyces yeasts.

Leducq et al. (2014) investigated the genetic and evolutionary basis of self-incompatibility in the plant tribe Biscutelleae (Brassicaceae), which has a sporophytic self-incompatibility system (SSI) in which the pollen (male gamete) phenotype is determined by the diploid genotype of the paternal plant. The pollen typically expresses cysteine-rich proteins (SCR) that are thought to interact with a transmembrane receptor (SRK) produced on the pistil that receives the pollen. The $S C R$ and $S R K$ genes are often genetically linked and called the $S$ locus. Successful fertilization depends on different alleles being present at the $S$ locus of pollen and pistils. Leducq et al. (2014) characterized SRK-like (SRKL) sequences in Biscutella neustriaca individuals from eight phenotypic incompatibility groups and discuss the association between SRKL sequences and self-incompatibility phenotypes and the similarities and differences between Biscutella and the better-studied but distantly related Brassicaceae such as Brassica and Arabidopsis.

\section{Sex Chromosomes Appear and Disappear and Have Different Rates of Natural Selection}

Sex chromosomes spend different amounts of time in males and females during an organism's evolution, usually experience less recombination than autosomes, and are often subject to dosage compensation. These differences lead to different selective pressures acting on sex-linked genes compared to the autosomal ones. Taken further, as essential genes move from the sex chromosome to autosomes, sex chromosomes can be lost and replaced with new ones that evolve from ancestral autosomes.

Blackmon and Demuth (2014) used phylogenetic analysis to reveal the tempo of $\mathrm{Y}$ chromosome turnover in Coleoptera. They analyzed karyotypes of 4724 beetle species that have heterogametic males that are $\mathrm{XY}, \mathrm{XO}$, or $\mathrm{Xy}+$. Xy+ are sex chromosome bivalents that display distance pairing (no synapsis) and thus do not recombine, allowing the authors to distinguish how differences in sex chromosome pairing and meiotic recombination influence the evolution of the sex chromosomes in two major beetle lineages, Adephaga (which have only XY and XO species), and Polyphaga (where over half of all species are $\mathrm{Xy}+$ ). Blackmon and Demuth (2014) find that in Adephaga, new Y chromosomes are evolving at the same rate as they are lost, whereas in Polyphaga Y chromosomes are twice as readily gained as lost. The authors propose that different meiotic mechanisms that evolve to ensure sex chromosome segregation affect the tempo of $\mathrm{Y}$ chromosome gain and loss.

Many sex chromosome pairs have a recombining region with largely similar gene content [pseudoautosomal region (PAR)]. Kirkpatrick and Guerrero (2014) use PAR to study sex-antagonistic (SA) selection. SA selection acts on alleles that are beneficial in one sex but detrimental to the other. Sex chromosomes tend to bear more SA alleles than autosomes, presumably due to their sex-specific inheritance and limited recombination. This study uses coalescent models to show that SA selection, together with limited recombination, should lead to different patterns of neutral genetic variation on the $\mathrm{X}$ and $\mathrm{Y}$ (or $\mathrm{Z}$ and $\mathrm{W}$ ) chromosomes. Importantly, these differences can be used to quantify the strength of SA selection. The authors test their model in Silene latifolia, a plant with young recombining sex chromosomes, to illustrate their approach.

\section{Different Molecular Mechanisms Operate in Male and Female Gametes}

The process of meiosis ensures that haploid complements of chromosomes segregate to each gamete through the processes of chromosome pairing, synapsis, and recombination. Given that sex chromosomes are either not homologous along their entire length (XY, ZW) or hemizygous (XO, $\mathrm{ZO}$ ), additional mechanisms have evolved to ensure their segregation during meiosis. While homology-dependent mechanisms function in heterogametic organisms, it is not known how meiosis is altered in the hemizygous sex to ensure segregation of the single sex chromosome. Furthermore, male and female gametes are morphologically distinct and undergo meiosis at different times during their developmental trajectories.

Checchi et al. (2014) examined the mechanisms that ensure segregation of the hemizygous $\mathrm{X}$ chromosome in C. elegans males (XO). They demonstrate that double-strand DNA breaks (DSB) are important for segregation of the 
hemizygous $\mathrm{X}$ and present evidence that DSBs could mediate sister chromatid synapsis (pseudosynapsis), instead of the typical synapses formed between homologs. They go further and show that different genes function to provide feedback on autosomal synapsis and DSB repair pathways. They posit that these fundamental differences in meiotic processes evolved to accommodate hemizygosity of the $\mathrm{X}$ chromosome.

Zhang et al. (2014) examined the transcriptomes and proteomes during the differentiation of maize anthers, a model system well suited for this study because of the relative ease of collecting tissues at different developmental stages. In angiosperms, the germline arises from somatic flower cells through a switch from mitotic proliferation to meiosis. Zhang et al. (2014) examined several stages on this developmental trajectory, from initial specification to final germinal and somatic cell differentiation. They describe transcriptome composition at different stages and show that many genes with meiotic functions are expressed prior to the onset of germ cell differentiation and meiosis. Consistent with recent findings in other systems, they also find a low correlation between transcript abundance and protein abundance, suggesting a major role of post-transcriptional regulation in this developmental process.

The inaugural collection highlights only some areas of sex-specific biology; there are many remaining questions and discoveries to be made on a broad range of topics. This growing resource will illuminate the myriad ways in which sex differences influence biological processes.

\section{Acknowledgments}

The authors thank participants of the Drosophila sex workshop, their laboratory members and colleagues for intellectual input over the years. MNA thanks O. Aparicio for comments on this perspective. The authors also acknowledge their grant support from National Institutes of Health, National Science Foundation, and US-Israel Binational Science Foundation. Finally, GENETICS and G3: Genes|Genomes|Genetics thank Deborah Charlesworth for her service as the Genetics of Sex collection editor.

\section{Literature Cited}

Barrett, S. C. H., 2002 The evolution of plant sexual diversity. Nat. Rev. Genet. 3: 274-284.

Blackmon, H., and J. P. Demuth, 2014 Estimating tempo and mode of Y chromosome turnover: explaining Y chromosome loss with the fragile Y hypothesis. Genetics 197: 561-572.

Charlesworth, D., and J. E. Mank, 2010 The birds and the bees and the flowers and the trees: lessons from genetic mapping of sex determination in plants and animals. Genetics 186: 9-31.

Checchi, P. M., K. S. Lawrence, M. V. Van, B. J. Larson, and J. Engebrecht, 2014 Pseudosynapsis and decreased stringency of meiotic repair pathway choice on the hemizygous sex chromosome of Caenorhabditis elegans males. Genetics 197: 543560.

Cline, T. W., and B. J. Meyer, 1996 Vive la difference: males vs. females in flies vs. worms. Annu. Rev. Genet. 30: 637-702.

Gamble, T., and D. Zarkower, 2012 Sex determination. Curr. Biol. 22: R257-R262.

Hughes, J. F., and S. Rozen, 2012 Genomics and genetics of human and primate Y chromosomes. Annu. Rev. Genomics Hum. Genet. 13(13): 83-108.

Kirkpatrick, M., and R. F. Guerrero, 2014 Signatures of sexantagonistic selection on recombining sex chromosomes. Genetics 197: 531-541.

Leduca, J., C. C. Gosset, R. Gries, K. Calin, E. Schmitt et al., 2014 Self-incompatibility in Brassicaceae: identification and characterization of SRK-like sequences linked to the S-locus in the tribe Biscutelleae. G3 4: 983-992.

Marin, I., and B. S. Baker, 1998 The evolutionary dynamics of sex determination. Science 281: 1990-1994.

Ni, M., M. Feretzaki, S. Sun, X. Wang, and J. Heitman, 2011 Sex in fungi. Annu. Rev. Genet. 45: 405-430.

Solieri, L., T. Chand Dakal, P. Giudici, and S. Cassanelli, 2014 Sex-determination system in the diploid yeast Zygosaccharomyces sapae. G3 4: 1011-1025.

Williams, T. M., and S. B. Carroll, 2009 Genetic and molecular insights into the development and evolution of sexual dimorphism. Nat. Rev. Genet. 10: 797-804.

Zarkower, D., 2001 Establishing sexual dimorphism: conservation amidst diversity? Nat. Rev. Genet. 2: 175-185.

Zhang, H., R. L. Egger, T. Kelliher, D. Morrow, J. Fernandes et al., 2014 Transcriptomes and proteomes define gene expression progression in pre-meiotic maize anthers. G3 4: 9931010.

Communicating editor: M. Johnston 